\title{
CANONICAL EQUIVARIANT EXTENSIONS USING CLASSICAL HODGE THEORY
}

\author{
CHRISTOPHER ALLDAY
}

Received 2 September 2004

Lin and Sjamaar have used symplectic Hodge theory to obtain canonical equivariant extensions for Hamiltonian actions on closed symplectic manifolds that have the strong Lefschetz property. Here we obtain canonical equivariant extensions much more generally by means of classical Hodge theory.

\section{Introduction}

In [4], Lin and Sjamaar show how to use symplectic Hodge theory to obtain canonical equivariant extensions of closed forms in Hamiltonian actions of compact connected Lie groups on closed symplectic manifolds which have the strong Lefschetz property. In this paper, we show how to do the same using classical Hodge theory. This has the advantage of applying far more generally. Our method makes use of Green's operator, but, as we will show in [3], it is often possible to make explicit calculations.

For nonabelian compact connected Lie groups, we use the small model, which is much simpler than the Cartan model and which has been shown to be chain homotopy equivalent to the Cartan model by Alekseev and Meinrenken (see [1]). In the abelian case, the two models are the same. The final section, however, considers the Cartan model.

\section{Notation and terminology}

Throughout this paper, $G$ will denote a compact connected Lie group with Lie algebra $\mathfrak{g}$; and $M$ will be a closed, connected, orientable, smooth manifold. $G$ will be acting on $M$; and $M$ will be given an invariant Riemannian metric. All cohomology will have real coefficients.

Definition 2.1. Let $i: M \rightarrow M_{G}$ be the inclusion of a fibre in the Borel construction bundle $M_{G} \rightarrow B G . M$ (or the action) is said to have a cohomology extension of the fibre (CEF) if

$$
i^{*}: H_{G}^{*}(M ; \mathbb{R}) \longrightarrow H^{*}(M ; \mathbb{R})
$$

is surjective. 
Remarks 2.2. "CEF" may also stand for "cohomologically extendable from the fibre" or "cohomological extendability of the fibre." Often "totally nonhomologous to zero" (TNHZ) has been used for this condition. It implies that $H_{G}^{*}(M ; \mathbb{R})$ is a free $H^{*}(B G ; \mathbb{R})$ module. And, when $G$ is a torus, CEF implies that

$$
\varphi^{*}: H_{G}^{*}(M ; \mathbb{R}) \longrightarrow H_{G}^{*}\left(M^{G} ; \mathbb{R}\right)
$$

is injective, where $\varphi: M^{G} \rightarrow M$ is the inclusion of the fixed point set $M^{G}$. The injectivity follows from the localization theorem of Borel, Hsiang, and Quillen. (See, e.g., [2].) And the injectivity of $\varphi^{*}$ is sometimes expressed by saying that $M$ is (cohomologically) equivariantly formal (CEF).

The purpose of this paper is to give a canonical section of $i^{*}$ in the CEF case. The method is an easy application of classical Hodge theory.

Using the invariant Riemannian metric, we define the Hodge star operator $*$ on $\Omega(M)$, and then $d^{*}$, the Laplacian (or Laplace-Beltrami) operator $\Delta$, and Green's operator G. (It should be clear from the context when $G$ is the Lie group and when $G$ is Green's operator.) Since the metric is invariant, $*, d^{*}, \Delta$, and $G$, like $d$, restrict to operators on the invariant forms $\Omega_{\text {inv }}(M)=\Omega(M)^{G}$. Thus the usual Hodge decomposition theorem (see, e.g., [5, Theorem 6.8], or [6, Chapter IV, Theorem 5.2]) applies to $\Omega_{\text {inv }}(M)$ without alteration.

The cohomology of the classifying space $H^{*}(B G ; \mathbb{R})$ is a polynomial ring: $H^{*}(B G ; \mathbb{R})=$ $\mathbb{R}\left[t_{1}, \ldots, t_{r}\right]$, where each $t_{j}$ has positive even degree and $r$ is the rank of $G$. We will often denote this ring by $R_{G}$. When $G$ is a torus, each $t_{j}$ has degree 2 ; and $R_{G}$ can be identified with the polynomial ring (symmetric algebra) on the dual of the Lie algebra of $G$, that is, $R_{G}=S\left(\mathfrak{g}^{*}\right)$. More generally, $R_{G}=S\left(\mathfrak{g}^{*}\right)^{G}$, the ring of invariants under the dual of the adjoint action.

The small model for computing the equivariant cohomology $H_{G}^{*}(M, \mathbb{R})$ is $R_{G} \otimes$ $\Omega_{\text {inv }}(M)$ with differential

$$
d_{G}=I \otimes d-\partial
$$

where $\partial=\sum_{j=1}^{r} t_{j} \otimes i_{j}$; and, for each $j, 1 \leq j \leq r, i_{j}$ is an operator on $\Omega_{\text {inv }}(M)$ of degree $-\operatorname{deg}\left(t_{j}\right)+1$.

To describe each $i_{j}$ in more detail, one considers $\wedge(\mathfrak{g})^{G}$, the subalgebra of the exterior algebra on $\mathfrak{g}$ fixed by the adjoint action. $\wedge(\mathfrak{g})^{G}$ is $\wedge(\mathscr{P})$, the exterior algebra on the primitives, and it can be identified with $H_{*}(G ; \mathbb{R})$. For a chosen basis $\left\{c_{1}, \ldots, c_{r}\right\}$ of $\mathscr{P}$, let $\left\{s_{1}, \ldots, s_{r}\right\}$ be the dual basis of generators of $H^{*}(G ; \mathbb{R})$, which is also an exterior algebra. Then $t_{j}$ corresponds to $s_{j}$ under transgression in the universal principal bundle $E G \rightarrow B G$. In the formula for $\partial, i_{j}$ is the inner product by the multivector field induced on $M$ by $c_{j}$ via the exponential map and the group action in the usual way. When $G$ is a torus, all $c_{j}$ have degree one, and the small model is the same as the Cartan model. When $G$ is nonabelian, however, it is far from obvious that the small model correctly computes $H_{G}^{*}(M ; \mathbb{R})$, that it does indeed do so is a theorem of Alekseev and Meinrenken (see [1]). Also see [1] for more details of the construction of the small model. 
Definition 2.3. In the small model, $R_{G} \otimes \Omega_{\text {inv }}(M)$, let

$$
P=\left(I \otimes d^{*} G\right) \partial
$$

where $G$ here is Green's operator.

So $P$ is an operator of degree zero. We will usually abbreviate $I \otimes d$ and $I \otimes d^{*} G$ simply as $d$ and $d^{*} G$; and so $d_{G}=d-\partial$ and $P=d^{*} G \partial$.

\section{The circle case}

In this short section, we give canonical equivariant extensions when $G=S^{1}$. This case is simpler than the general case and nicely illustrates the method. The small model is the same as the Cartan model in this case, namely, $R_{G} \otimes \Omega_{\text {inv }}(M)$; and $R_{G}=\mathbb{R}[t]$, where $\operatorname{deg}(t)=2$. Also $\partial=t \otimes i_{V}$, where $V$ is the vector field coming from the circle action. The case rests on the following lemma.

Lemma 3.1. Assume that $M$ has a CEF. (See Definition 2.1.) Let $\alpha \in \Omega_{\mathrm{inv}}(M)$, and suppose that $d i_{V}(\alpha)=0$. Then $i_{V}(\alpha)=d \beta$ for some $\beta \in \Omega_{\text {inv }}(M)$.

Proof. Since $d i_{V}(\alpha)=0, d_{G} i_{V}(\alpha)=0$. Let $x=\left[i_{V}(\alpha)\right]_{G}$, the equivariant cohomology class of $i_{V}(\alpha)$. So $x$ restricts to zero on the fixed point set $M^{G}$, which is nonempty by CEF. So $x=0$, again by CEF. (See Remarks 2.2 following Definition 2.1.) Hence $i^{*}(x)=0$, where $i: M \rightarrow M_{G}$ is the inclusion of a fibre. Thus, $i_{V}(\alpha)=d \beta$ for some $\beta \in \Omega_{\text {inv }}(M)$.

Theorem 3.2. Assume that $M$ has a CEF. Let $\alpha \in \Omega_{\text {inv }}(M)$ be a closed form (i.e., $d \alpha=0$ ). Let

$$
\widehat{\alpha}=(I-P)^{-1}(\alpha)=\alpha+P(\alpha)+P^{2}(\alpha)+\cdots+P^{n}(\alpha)+\cdots
$$

$\left(P^{m}(\alpha)=0\right.$ for all $m$ such that $\left.2 m>\operatorname{deg}(\alpha)\right)$. Then $d_{G}(\hat{\alpha})=0$.

Hence the map $\alpha \mapsto[\hat{\alpha}]_{G}$, restricted to harmonic forms, is a canonical cohomology extension of the fibre $H^{*}(M ; \mathbb{R}) \rightarrow H_{G}^{*}(M ; \mathbb{R})$.

Proof. $d_{G} \alpha=-\partial \alpha=-t i_{V}(\alpha)$, where we have abbreviated $t \otimes i_{V}$ as $t i_{V}$. And $d i_{V}(\alpha)=$ $-i_{V} d(\alpha)=0$. Hence, by Lemma 3.1, $i_{V}(\alpha)$ is a boundary. So $i_{V}(\alpha)=d d^{*} G i_{V}(\alpha)$. Now $d_{G}(\alpha+P(\alpha))=-t i_{V}(\alpha)+d P(\alpha)-t i_{V} P(\alpha)=-t i_{V} P(\alpha)$.

Inductively, suppose that $d_{G}\left(\alpha+P(\alpha)+\cdots+P^{j}(\alpha)\right)=-t i_{V} P^{j}(\alpha)$. Then $d i_{V} P^{j}(\alpha)=$ $-i_{V} d P^{j}(\alpha)=-i_{V} \partial P^{j-1}(\alpha)=0$. So, again by Lemma 3.1, $i_{V} P^{j}(\alpha)$ is a boundary; and so $i_{V} P^{j}(\alpha)=d d^{*} G i_{V} P^{j}(\alpha)$. Hence $d_{G}\left(\alpha+P(\alpha)+\cdots+P^{j}(\alpha)+P^{j+1}(\alpha)\right)=-t i_{V} P^{j+1}(\alpha)$.

Example 3.3. Suppose that $M$ is symplectic and that the action is Hamiltonian. Let $\omega \in$ $\Omega_{\text {inv }}(M)$ be the symplectic form, and let $\mu$ be the moment map. So $d \mu=i_{V}(\omega)$. Suppose, further, that $\mu$ has been chosen so that its average value is zero. Thus, in the Hodge decomposition, the harmonic part of $\mu$ is zero; and so $\mu=d^{*} d G(\mu)=d^{*} G d(\mu)$. So $P(\omega)=t \mu$; and $\widehat{\omega}=\omega+t \mu$, the usual equivariant extension of $\omega$. 


\section{The small model}

In this section, $G$ is any compact connected Lie group. Using the small model, the main result looks the same.

Theorem 4.1. Assume that $M$ has a CEF. Let $\alpha \in \Omega_{\text {inv }}(M)$ be a closed form (i.e., $d \alpha=0$ ). Let

$$
\widehat{\alpha}=(I-P)^{-1}(\alpha)=\alpha+P(\alpha)+P^{2}(\alpha)+\cdots+P^{n}(\alpha)+\cdots .
$$

Then $d_{G}(\hat{\alpha})=0$. Hence, the map $\alpha \mapsto[\hat{\alpha}]_{G}$, restricted to harmonic forms, is a canonical cohomology extension of the fibre $H^{*}(M ; \mathbb{R}) \rightarrow H_{G}^{*}(M ; \mathbb{R})$.

Proof. The localization theorem (in a useful form) is not valid for nonabelian $G$. We will compensate for this by using induction not only on the power of $P$ but also on the degree of $\alpha$.

First, $d_{G}(\alpha)=-\partial \alpha$. By CEF, $\alpha$ has an equivariant extension; and so $\partial \alpha$ is a $d$-boundary. Hence $\partial \alpha=d P(\alpha)$. Since $\partial P(\alpha)=0$ if $\operatorname{deg}(\alpha) \leq 2$, this starts both inductions.

Now suppose that $d_{G}\left(\alpha+P(\alpha)+\cdots+P^{j}(\alpha)\right)=-\partial P^{j}(\alpha)$. For $I=\left(i_{1}, \ldots, i_{j+1}\right)$, where $1 \leq i_{1} \leq \cdots \leq i_{j+1} \leq r=\operatorname{rank}(G)$, let $t_{I}=t_{i_{1}} \cdots t_{i_{j+1}}$. Collecting terms, we can set

$$
\partial P^{j}(\alpha)=\sum_{I} t_{I} \alpha_{I}
$$

where $\alpha_{I} \in \Omega_{\text {inv }}(M)$ and $\operatorname{deg}\left(\alpha_{I}\right)=\operatorname{deg}(\alpha)-\operatorname{deg}\left(t_{I}\right)+1$. So $\operatorname{deg}\left(\alpha_{I}\right)<\operatorname{deg}(\alpha)$.

Clearly, $d \partial P^{j}(\alpha)=0$. So, since the monomials $t_{I}$ are linearly independent, $d \alpha_{I}=0$ for all $I$. Thus, by the induction hypothesis on degree, we can assume that $d_{G}\left(\hat{\alpha}_{I}\right)=0$ for all I. On the other hand, for $i \geq 1$,

$$
\sum_{I} t_{I} P^{i}\left(\alpha_{I}\right)=P^{i} \sum_{I} t_{I} \alpha_{I}=P^{i} \partial P^{j}(\alpha)=0,
$$

because $P \partial=d^{*} G \partial^{2}=0$. Thus,

$$
\sum_{I} t_{I} \hat{\alpha}_{I}=\sum_{I} t_{I} \alpha_{I}=-d_{G}\left(\alpha+P(\alpha)+\cdots+P^{j}(\alpha)\right) .
$$

Hence $\sum_{I} t_{I}\left[\hat{\alpha}_{I}\right]_{G}=0$.

Since $H_{G}^{*}(M ; \mathbb{R})$ is a free $R_{G}$-module by CEF, it follows that $i^{*}\left[\hat{\alpha}_{I}\right]_{G}=0$ for all $I$. (As above, $i: M \rightarrow M_{G}$ is the inclusion of a fibre. For details, see Remark 4.2(1) below.) Thus each $\alpha_{I}$ is a boundary; and so $\partial P^{j}(\alpha)=d P^{j+1}(\alpha)$.

Remarks 4.2. (1) The details of the free module argument are as follows. By CEF, there are $a_{1}, \ldots, a_{k}$ in $H_{G}^{*}(M ; \mathbb{R})$ such that $\left\{a_{1}, \ldots, a_{k}\right\}$ is a basis for $H_{G}^{*}(M ; \mathbb{R})$ as a free $R_{G}$-module, and $\left\{i^{*}\left(a_{1}\right), \ldots, i^{*}\left(a_{k}\right)\right\}$ is a basis for $H^{*}(M ; \mathbb{R})$ as a vector space. Let $\left[\hat{\alpha}_{I}\right]_{G}=\sum_{j} \lambda_{I j} a_{j}+b_{I}$, where $\lambda_{I j} \in \mathbb{R}$ and $b_{I}$ is a sum of terms of positive degree in $t_{1}, \ldots, t_{r}$. Since $\sum_{I} t_{I}\left[\hat{a}_{I}\right]_{G}=$ $0, \sum_{I, j} \lambda_{I j} t_{I} a_{j}=0$, because all the $t_{I}$ 's have the same polynomial degree (although not necessarily the same total degree). Hence, each $\lambda_{I j}=0$. So $i^{*}\left[\hat{\alpha}_{I}\right]_{G}=0$ for each $I$. (In effect, for the purpose of this argument, we regrade $R_{G}$ so that $t_{1}, \ldots, t_{r}$ all have the same degree, as in the torus case.) 
(2) We will give another proof of Theorem 4.1 in [3] using the minimal Hirsch-Brown model.

(3) In [1], Alekseev and Meinrenken give a canonical embedding of the small model into the Cartan model which is a homotopy equivalence of differential $R_{G}$-modules. Applying this mapping to $\hat{\alpha}$ gives a canonical equivariant extension of $\alpha$ in the Cartan model. In the next section, however, following suggestions by Alekseev and Meinrenken, we obtain a version of Theorem 4.1 for the Cartan model directly.

\section{The Cartan model}

Again, in this section, $G$ is any compact connected Lie group; but here, we use the Cartan model instead of the small model. Thus, in the definition of $P$, the operator $\partial$ is now that of the Cartan model. The methods of this section, in particular Lemmas 5.3 and 5.4, are entirely due to Alekseev and Meinrenken.

Again, the theorem looks the same.

Theorem 5.1. Assume that $M$ has a CEF. Let $\alpha \in \Omega_{\text {inv }}(M)$ be a closed form (i.e., $d \alpha=0$ ). Let

$$
\widehat{\alpha}=(I-P)^{-1}(\alpha)=\alpha+P(\alpha)+P^{2}(\alpha)+\cdots+P^{n}(\alpha)+\cdots
$$

Then $d_{G}(\hat{\alpha})=0$. Hence, the map $\alpha \mapsto[\hat{\alpha}]_{G}$, restricted to harmonic forms, is a cohomology extension of the fibre $H^{*}(M ; \mathbb{R}) \rightarrow H_{G}^{*}(M ; \mathbb{R})$.

The theorem will follow directly from Lemma 5.4. First, however, note that it is well known that the inclusion of cochain complexes $\left(\Omega_{\mathrm{inv}}(M), d\right) \rightarrow(\Omega(M), d)$ induces an isomorphism in cohomology. It is only a little less well known that the inclusion of cochain complexes

$$
\left(S\left(\mathfrak{g}^{*}\right)^{G} \otimes \Omega(M)^{G}, d\right) \longrightarrow\left(\left(S\left(\mathfrak{g}^{*}\right) \otimes \Omega(M)\right)^{G}, d\right)
$$

also induces an isomorphism in cohomology, $H^{*}(B G ; \mathbb{R}) \otimes H^{*}(M ; \mathbb{R}) .\left(\Omega(M)^{G}=\right.$ $\Omega_{\text {inv }}(M)$.) Thus we have the next lemma.

Lemma 5.2. Let $a \in\left(S^{p}\left(\mathfrak{g}^{*}\right) \otimes \Omega^{q}(M)\right)^{G}$ and suppose that $d a=0$. Then there is $b \in$ $S^{p}\left(\mathfrak{g}^{*}\right)^{G} \otimes \Omega^{q}(M)^{G}$ and $c \in\left(S^{p}\left(\mathfrak{g}^{*}\right) \otimes \Omega^{q-1}(M)\right)^{G}$ such that $a=b+d c$.

The next lemma shows that CEF implies the existence of more general equivariant extensions.

Lemma 5.3. Assume that $M$ has a CEF. Let $a \in\left(S^{p}\left(\mathfrak{g}^{*}\right) \otimes \Omega^{q}(M)\right)^{G}$, and suppose that da= 0 . Then there are $a_{j} \in\left(S^{p+j}\left(\mathfrak{g}^{*}\right) \otimes \Omega^{q-2 j}(M)\right)^{G}$ for $j \geq 0$, such that $a_{0}=a$ and $d a_{j}=\partial a_{j-1}$ for all $j \geq 1$. Thus $d_{G}\left(a+a_{1}+\cdots+a_{j}+\cdots\right)=0$.

Proof. Let $a=b+d c$ as in Lemma 5.2. Then CEF clearly implies the existence of $b_{j} \in$ $\left(S^{p+j}\left(\mathfrak{g}^{*}\right) \otimes \Omega^{q-2 j}(M)\right)^{G}$ for $j \geq 0$, such that $b_{0}=b$ and $d b_{j}=\partial b_{j-1}$ for all $j \geq 1$. Now put $a_{1}=b_{1}-\partial c$, and put $a_{j}=b_{j}$ for $j \geq 2$. 
The final lemma, which easily implies Theorem 5.1, shows that arbitrary partial equivariant extensions can always be extended indefinitely (assuming CEF).

Lemma 5.4. Assume that $M$ has a CEF. Let $a \in\left(S^{p}\left(\mathfrak{g}^{*}\right) \otimes \Omega^{q}(M)\right)^{G}$, and suppose that $d a=$ 0 . Suppose given, for $0 \leq j \leq m, a_{j} \in\left(S^{p+j}\left(\mathfrak{g}^{*}\right) \otimes \Omega^{q-2 j}(M)\right)^{G}$ such that $a_{0}=a$ and $d a_{j}=$ $\partial a_{j-1}$ for $1 \leq j \leq m$. Then there is $a_{m+1} \in\left(S^{p+m+1}\left(\mathfrak{g}^{*}\right) \otimes \Omega^{q-2 m-2}(M)\right)^{G}$ such that d $a_{m+1}=$ $\partial a_{m}$. In particular, one could take $a_{m+1}=P\left(a_{m}\right)$.

Proof. We use induction on $m$. The case $m=0$ is clear by Lemma 5.3. Also by Lemma 5.3, for $j \geq 1$, there are $y_{j} \in\left(S^{p+j}\left(\mathfrak{g}^{*}\right) \otimes \Omega^{q-2 j}\right)^{G}$ such that $d y_{1}=\partial a$, and $d y_{j}=\partial y_{j-1}$ for all $j \geq 2$. For $1 \leq j \leq m$, let $c_{j}=a_{j}-y_{j}$. Then $d c_{1}=0$, and for $2 \leq j \leq m, d c_{j}=\partial c_{j-1}$. Thus, by the induction hypothesis, there is $c_{m+1}$ such that $d c_{m+1}=\partial c_{m}$. Now put $a_{m+1}=$ $c_{m+1}+y_{m+1}$.

Remark 5.5. The analog of Lemma 5.4 for the small model follows directly from the proof of Theorem 4.1. And, thanks to Lemma 5.2, Theorem 5.1 could be proved along lines similar to those used to prove Theorem 4.1. Equally, of course, Theorem 4.1 could be proven by the methods of this section.

\section{Acknowledgment}

I would like to thank A. Alekseev and E. Meinrenken for many helpful discussions. In particular, the last section is due to them.

\section{References}

[1] A. Alekseev and E. Meinrenken, Equivariant cohomology and the Maurer-Cartan equation, 2004, to appear in Duke Math. J.

[2] C. Allday and V. Puppe, Cohomological Methods in Transformation Groups, Cambridge Studies in Advanced Mathematics, vol. 32, Cambridge University Press, Cambridge, 1993.

[3] - The minimal Hirsch-Brown model via classical Hodge theory, 2004, to appear in Pacific J. Math.

[4] Y. Lin and R. Sjamaar, Equivariant symplectic Hodge theory and the $d_{G} \delta$-lemma, J. Symplectic Geometry 2 (2004), 267-278.

[5] F. W. Warner, Foundations of Differentiable Manifolds and Lie Groups, Graduate Texts in Mathematics, vol. 94, Springer, New York, 1983.

[6] R. O. Wells Jr., Differential Analysis on Complex Manifolds, Graduate Texts in Mathematics, vol. 65, Springer, New York, 1980.

Christopher Allday: Department of Mathematics, University of Hawaii, 2565 McCarthy Mall, Honolulu, HI 96822-2273, USA

E-mail address: chris@math.hawaii.edu 


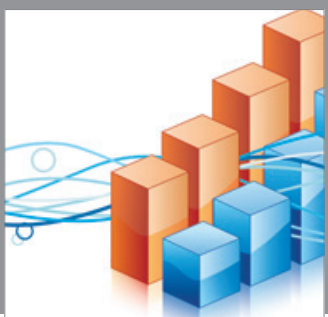

Advances in

Operations Research

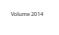

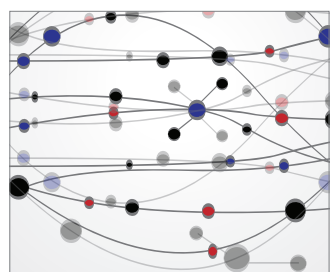

\section{The Scientific} World Journal
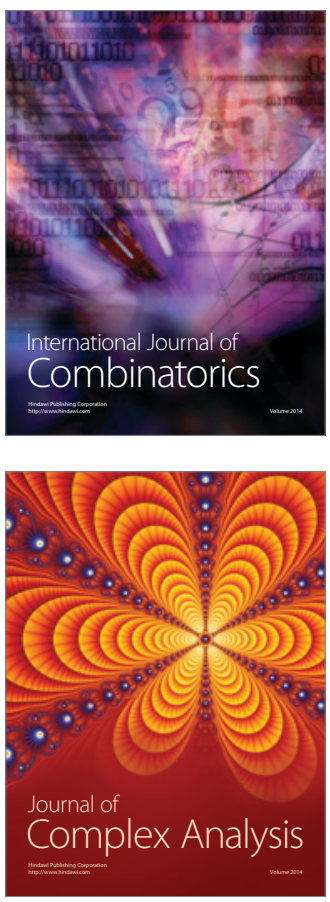

International Journal of

Mathematics and

Mathematical

Sciences
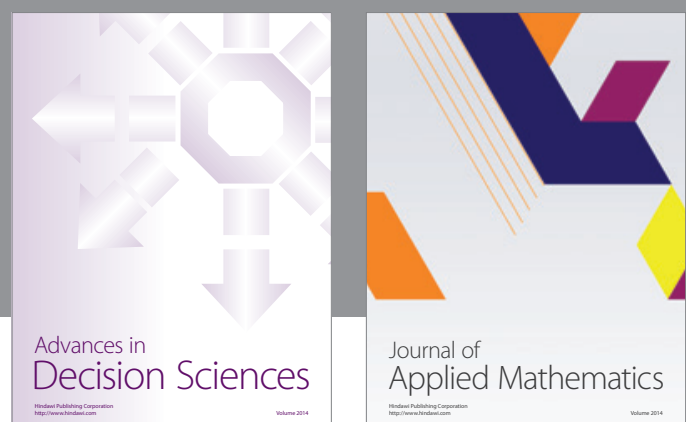

Journal of

Applied Mathematics
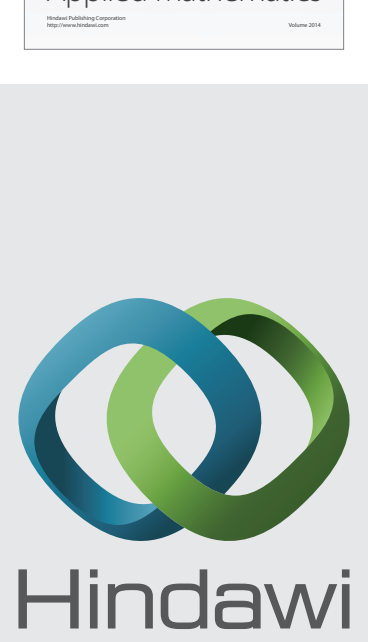

Submit your manuscripts at http://www.hindawi.com
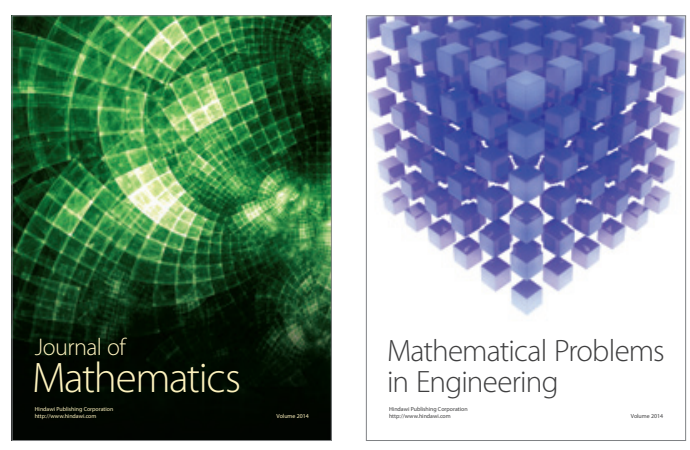

Mathematical Problems in Engineering
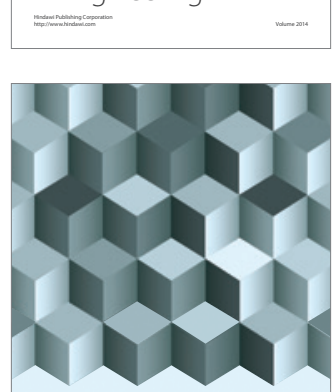

Journal of

Function Spaces
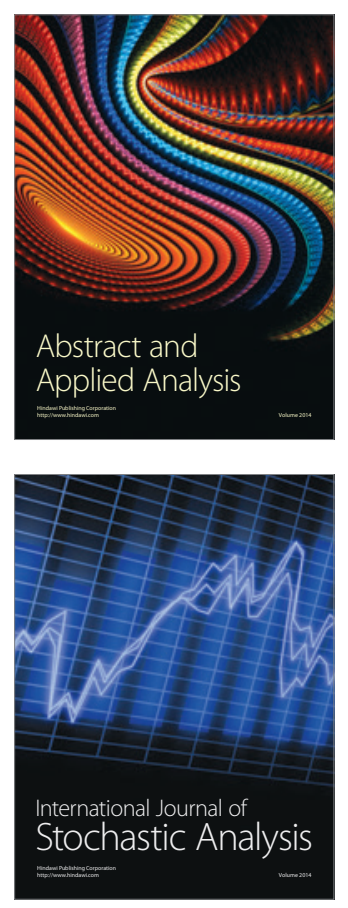

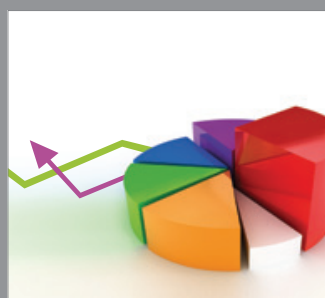

ournal of

Probability and Statistics

Promensencen
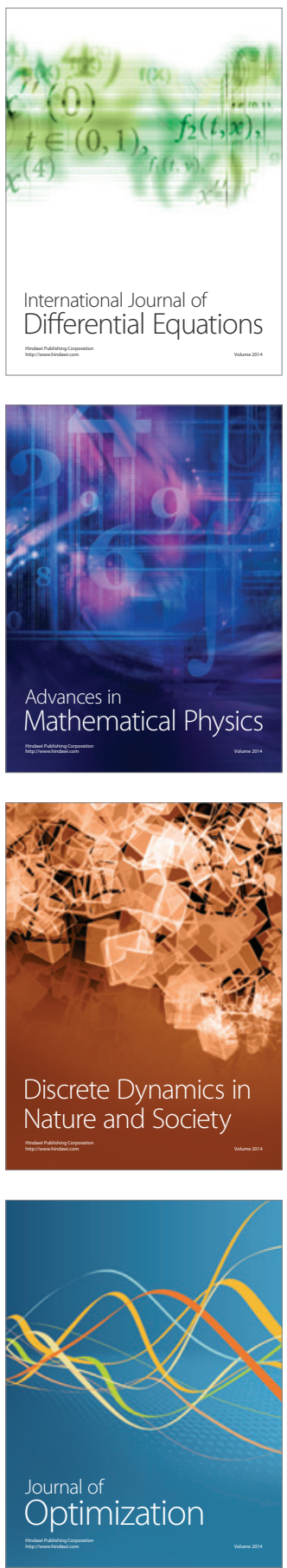